\title{
Is there a space-time continuum in olfaction?
}

\author{
Michael Leon · Brett A. Johnson
}

Received: 2 December 2008/Revised: 18 February 2009/Accepted: 23 February 2009/Published online: 18 March 2009

(C) The Author(s) 2009. This article is published with open access at Springerlink.com

\begin{abstract}
The coding of olfactory stimuli across a wide range of organisms may rely on fundamentally similar mechanisms in which a complement of specific odorant receptors on olfactory sensory neurons respond differentially to airborne chemicals to initiate the process by which specific odors are perceived. The question that we address in this review is the role of specific neurons in mediating this sensory system - an identity code-relative to the role that temporally specific responses across many neurons play in producing an olfactory perception-a temporal code. While information coded in specific neurons may be converted into a temporal code, it is also possible that temporal codes exist in the absence of response specificity for any particular neuron or subset of neurons. We review the data supporting these ideas, and we discuss the research perspectives that could help to reveal the mechanisms by which odorants become perceptions.
\end{abstract}

Keywords Olfactory coding - Identity code .

Temporal code $\cdot$ Odor perception $\cdot$ Sensory coding

\section{Introduction}

In his seminal electrophysiological studies of olfactory bulb neurons, Adrian [1] recognized that odorants evoked both spatial and temporal patterns of neural activity, and he speculated that information relevant to odor perception might be contained in either or both types of pattern. In the following decades, the combined "spatiotemporal" nature

M. Leon $(\bowtie) \cdot$ B. A. Johnson

Department of Neurobiology and Behavior, University of California Irvine, Irvine, CA 92697-4550, USA

e-mail: mleon@uci.edu of the neural coding of odor perceptions was widely assumed, and while individual researchers characterized both types of response patterns, they did not actually provide a model to explain the relationships between these patterns and odor perception.

Recently, many publications have described separate lines of research involving either spatial or temporal patterns of olfactory response. For example, wellcharacterized spatially distinct patterns of activity are evoked reliably by numerous odorants in the glomerular layer of the rat (Rattus norvegicus) olfactory bulb, where activity occurs in functional domains responding to particular aspects of odorant chemistry [2, 3]. Moreover, the overall glomerular response patterns accurately predict odor perceptions without any reference to temporal activity patterns [4]. These findings, as well as similar findings in zebrafish (Danio danio), catfish (Ictalurus punctatus and Ameiurus nebulosus), fruit flies (Drosophila melanogaster), and honeybees (Apis mellifera) [5-11], have led to the suggestion that the identity of the activated neurons might carry the bulk of the information about perceived odor [3]. A parallel development in the field of olfactory coding has involved mathematical analyses of the temporal aspects of neural activity, and these studies have led to models of odor coding that are based on temporal patterns of activity within highly distributed neural ensembles. Although the identity of the neurons displaying the complex temporal patterns is considered, the models do not include any special involvement of particular neurons in the system to encode information about any given odor [12-15].

Is it possible to include both the identity/spatial coding and temporal coding notions of olfactory processing into a common framework, given that researchers working with either model typically make no more than a passing reference to the other? Is there a transformation of 
information from specific activated neurons at one level of processing to specific temporal patterns that convey the information required for perception at a higher level of processing? Or are the temporal patterns simply an epiphenomenon that accompanies the spatially distinct responses within the olfactory system?

\section{Spatial patterns of activity across glomeruli}

\section{Rodents}

There is a consensus that individual olfactory sensory neurons in rodents express one type of odorant receptor and that neurons expressing the same type exist within broad zones along the nasal epithelium [16-18]. Figure 1 diagrams the general organization of both the vertebrate and invertebrate olfactory systems. The axons of the homologous sensory neurons converge in their projections into glomeruli of the olfactory bulb of the brain $[19,20]$, and each glomerulus appears to receive projections only from sensory neurons expressing the same receptor [21], so that a representation of the activity levels of all glomeruli should allow a read-out of the activation of the full complement of receptors.

Shepherd et al. [22] first used the 2-deoxyglucose technique to reveal a map of differential responsiveness to odorants at the level of the glomerular layer in rats. We have continued this work by constructing average maps of activity levels across the entire glomerular layer after exposing different groups of rats to one of a large battery of monomolecular odorants that include both systematically related chemicals and chemically diverse compounds [3] (http://leonserver.bio.uci.edu). We found that chemically distinct odorants stimulate different glomeruli, thereby

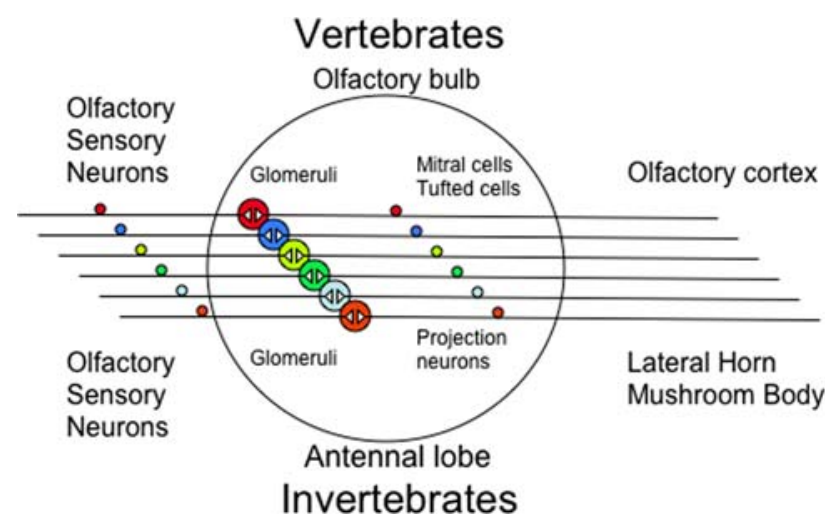

Fig. 1 This diagram shows the organization of the vertebrate and invertebrate olfactory systems. The olfactory sensory neurons express different olfactory receptors and homogeneous sensory neurons cluster in specific glomeruli. Second-order neurons then project to higher brain centers producing very different spatial activity patterns. On the other hand, odorants sharing molecular features such as functional groups, hydrocarbon structures, and/or overall properties such as water solubility, tend to stimulate overlapping sets of glomeruli. As a result of these overlaps, the glomerular layer can be partitioned into separate domains responding to odorants with particular molecular features [3] (Fig. 2). Some of these domains contain a finer organization, wherein odorants that are most similar in molecular length stimulate glomeruli that are located nearest one another in the domain [23-25]. This nearestneighbor patterning, together with the strong lateral inhibitory networks between glomeruli and mitral cell bulbar projection neurons [26, 27], offers an anatomical foundation for sharpening the responses of mitral cells [28]. The response sharpening also would have the effect of decorrelating the responses of mitral cells emanating from neighboring glomeruli with overlapping specificity. These data support the idea of an identity code for odor quality in which the identity of specific activated neurons is critical for processing the signal for odor quality. The systematic organization of the responses within the identified domains gives some support to the possibility that the actual spatial organization also plays a role in olfactory processing.

Importantly, quantitative similarities between spatial patterns of activity measured across the entire glomerular layer accurately predict similarities in perceived odors. This predictive relationship has been found using a variety of behavioral tests of perception, including cross-habituation of spontaneous responses to novel odors [29-31], numbers of trials needed to learn a discrimination between two odors [32, 33], and the number of errors committed in a five-odorant confusion matrix after extensive training with each odor [4] (Fig. 3). The predictive nature of the spatially distinct patterns for odor-guided behavior evoked

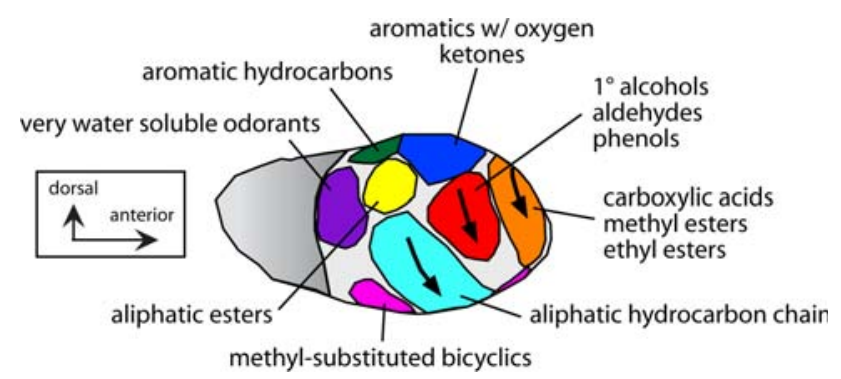

Fig. 2 The glomerular layer of the rat olfactory bulb is organized into domains containing clusters of glomeruli that respond best to odorants sharing molecular features or overall molecular properties as shown. The diagram is a summary of our results using the 2DG technique [3]. The lateral aspect of the olfactory bulb is shown. A similar organization is present on the medial surface of the bulb. Arrows indicate chemotopic progressions within three domains, where odorants of increasing molecular length activate progressively ventral glomeruli. This figure is modified from [3] 


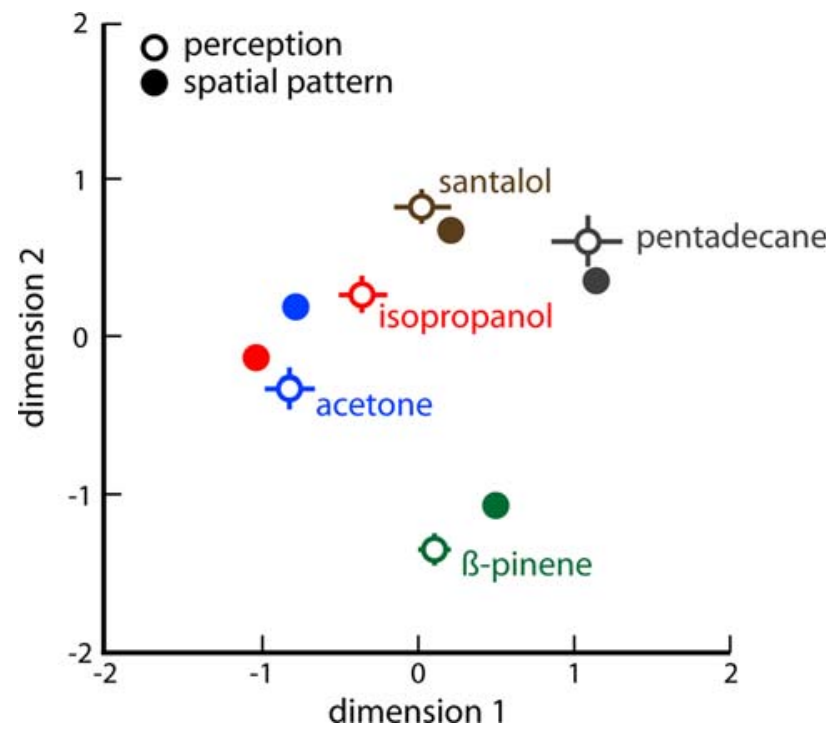

Fig. 3 Relative similarities in spatial patterns of glomerular layer activity are predictive of relative similarities in odors perceived by rats. Patterns of activity were measured using 2DG uptake, and perceptual similarities were measured using a five-odorant confusion matrix [4]. Relationships among both the patterns and the perceptions were visualized using multidimensional scaling. The perceptual data were reliably predictive across five separate animals as indicated by the standard errors. This figure is modified from [4]

by odorants provides clear support for the importance of the identity of specific neurons underlying olfactory processing.

Spatial representations of odorants in simpler olfactory systems

Because most of the systematic analysis related to temporal coding of odor information has been conducted on olfactory systems in insects and fish, it is useful to compare the organization of glomerular responses in these types of animals to those for rodents. In contrast to the more than 1,000 different functional receptors in rats and mice (Mus musculus) [18, 34], honeybees have about 160 receptors [35], zebrafish possess about 140 receptors [36], and fruit flies have about 60 receptors [37, 38]. Just as in rodents, distinct species-typical patterns of glomerular activity are evoked by distinct odorants in fruit flies, honeybees, and zebrafish $[5,6,9,10]$. The homologous nature of the sensory neuron input to each glomerulus has been shown in fruit flies [38].

In zebrafish and catfish, amino acids activate clusters of glomeruli that are spatially separated from other sets of glomeruli responding to other broad odorant chemical classes such as bile acids and nucleic acids [5, 6, 39], but the number of domains appears to be smaller in fish, and there is little evidence for a finer organization that would establish more detailed nearest-neighbor relationships between glomeruli within the domains $[5,6]$. In honeybees and fruit flies [9, 40], there is no clear domain organization with respect to odorant chemical structure for those odorants that have been studied. The clustering of responses to related chemicals into spatial domains that is observed in rat olfactory bulbs may reflect both a larger number of odorant receptors in that species and an evolutionary expedience to shorten the connections necessary for optimal decorrelation of responses by lateral inhibition. In insects, the corresponding structures may be small enough that this anatomical organization is not required. Indeed, there is evidence that differences in activity between projection neurons and their associated glomeruli in honeybees arise through inhibition by non-neighboring projection neurons that have related specificity [41]. Thus, the same functional principle may be served in honeybees and mammals, even though the anatomical organization is different. On the other hand, the absence of response clustering in honeybees and fruit flies may simply indicate species-specific differences in organization, given that moth antennal lobes contain distinct zones that respond to different odorant chemical classes [42].

In catfish, a chemotopic map is preserved from the glomerular layer to the mitral cell layer and also is observed in forebrain targets of the mitral cell projections [39, 43]. Unlike the observations in rats, catfish cortical neurons can be identified that respond to certain classes of amino acid odorants with a specificity that is very similar to that of bulbar projection neurons or even olfactory sensory neurons, both in terms of those odorants that are excitatory and those that are inhibitory, a finding that raises the possibility of a "labeled line" for information about some odorants in this species [7, 39, 43-46].

In agreement with what has been observed for rats, spatial patterns of activity in the honeybee antennal lobe accurately predict perceptions of odor as measured by the degree of generalization of a conditioned proboscis extension response [11]. Similar to the data that have been reported for rats, zebrafish and catfish have difficulties discriminating amino acid odorants that evoke very similar spatial patterns of activity in the zebrafish olfactory bulb [8]. In all of these species, the pattern of activity evoked by specific olfactory sensory neurons predicted the olfactory perception accurately, and these data therefore provide strong support for an identity code for odor quality.

Studies on the representation of odorant chemistry generally focus on monomolecular odorants, but olfactory systems are presented with complex odorant mixtures as their predominant stimuli. In invertebrates, the presence of multiple odorants in a mixture can result in interactions at many levels, and that has been elegantly demonstrated by reconstructing the responses of the lobster olfactory system 
to natural food stimuli [47]. Similar results for food-related mixtures have been obtained for catfish [48], and inhibitory interactions between odorants in arbitrary mixtures also were observed during optical imaging of calcium responses in honeybee and Drosophila glomeruli [49, 50]. Surprisingly, a few recent studies have reported that glomerular responses on the dorsal surface of the anesthetized rodent bulb can be predicted from responses to monomolecular components [51-53]. However, assessments of glomerular activity over the whole bulb in conscious rats have indicated that mixture interactions may be common in mammals as well $[54,55]$. It remains to be determined to what extent an understanding of these mixture interactions will inform our thinking about various proposed mechanisms of odor coding.

\section{Transformation of olfactory information beyond the glomeruli}

The fact that glomerular activity patterns predict perceptual relationships may indicate that the method of response mapping has successfully captured the bulk of the information about the stimulus that is coming into the system. If one has measured the relative levels of stimulation of all of the odorant receptors, one may in fact have all of the external information available to the nervous system to establish an odor perception. The predictive power of the spatial pattern of activity, however, does not necessarily indicate a mechanism through which odor information is then extracted. Indeed, the glomerular activity pattern may have more information than is available to higher levels of the olfactory system given that the discrete information about odorant chemistry that also is present at the level of the glomerular activation pattern does not appear to be fully available at higher levels of processing. The presence of an activity map related to odorant chemistry in the glomerular layer probably reflects the transformation of information about molecular features of odorants by way of spatial relationships that tune or decorrelate mitral cell responses to closely related odorants that are not well discriminated by individual odorant receptor proteins. Thus, the glomerular activity maps themselves probably do not constitute a code, but rather are a first step in the processing of odorants.

Because quantitative activity maps show a much greater response in some glomeruli than in others, the identity of the most active cells might convey considerable information. This information appears to be largely intact in the output cells of the bulb, the mitral cells, as judged by correlated 2DG responses in deeper layers that are postsynaptic to the mitral cells $[23,56]$, by the pattern of expression of immediate genes [57], by optical recording of postsynaptic responses in several species [9, 58, 59], as well as by physiological recordings from mitral cells either in regions of $2 \mathrm{DG}$ uptake $[60,61]$, or from neurons emanating from glomeruli that are known to be activated by optical imaging [62, 63].

In rodents, convergence of projections from many mitral cells in widely distributed bulbar locations onto any given pyramidal cell in the olfactory cortex may cause much of the information that is segregated in the bulb to be combined in the cortex [64]. Studies on the cortical projections of small numbers of mitral cells suggest a patchy, rather than a homogeneous connection, which might cause pyramidal cells to become more activated by certain combinations of mitral cell responses than by other combinations [65-67]. Thus, there may be either combinations of odorants in certain mixtures, or combinations of molecular features in certain individual odorant chemicals, that can produce a particular cortical response. Odorantevoked activity patterns measured by Fos-like immunohistochemistry in the anterior olfactory cortex showed widespread but patchy labeling that is fully consistent with the anatomical projections to the olfactory cortex [68, 69]. Responses recorded using electrophysiology in cortex also suggest a broader responsiveness of pyramidal cells than mitral cells [70, 71], although cross-habituation studies establish that the cortical response to each odorant can be even more narrowly tuned with respect to fine-scale differences in odorant chemistry than is the mitral cell response [72]. In cross-habituation studies, the investigator takes advantage of spontaneous responses to novel odorants. As an odor becomes less and less novel upon repeated presentation, the response decreases, and when a second odorant is introduced, the response resumes only if the odor is regarded differently from the first odorant.

In considering the specificity of the primary output neurons at the first stage of central olfactory processing (mitral cells or antennal lobe projection neurons), it is important first to define what comprises a meaningful response in these cells, and we would argue that the significance of a response should be determined by what the nervous system uses for the behaviorally relevant processing of olfactory information. In studies of temporal response patterns, projection neurons or mitral cells are recorded without knowing whether their dendrites are present in highly activated glomeruli or in glomeruli that do not respond differentially to the odorant. Although nearly all of the recorded neurons may change their activity in response to odorants, many respond after a long delay, and the change in their activity often involves phases of suppressed firing [73-77], response patterns that may be more likely observed for the neurons that extend dendrites into nonresponsive or poorly responsive glomeruli. If all of these changes in activity were lumped together when 
considering the odorant signal, then the high degree of specificity reflected in various glomerular imaging studies would appear to have been lost by the second-order neurons that report olfactory information to higher regions. On the other hand, if mitral cells associated with the most active glomeruli were monitored, it is possible that the coordinated early responses in this limited set of cells would contain the behaviorally significant information.

Although there is some temporal patterning of glomerular activity in the dorsal part of the mouse olfactory bulb, as revealed by optical recording techniques [78, 79], there are strong indications that most of the temporal patterning of mitral cell and projection neuron responses arises as a result of synaptic interactions within and between glomeruli and their associated projection neurons [26, 27, 58, 80, 81]. Indeed, the feedback and feedforward connections within the vertebrate olfactory bulb inevitably result in temporal patterning of activity involving large parts of the structure [82]. Because these same circuits may mediate the tuning of output responses to similar odorants, the existence of temporal patterning of projection neuron action potentials is not itself evidence for the use of temporal information in odor processing. Indeed, the temporal patterns conceivably could arise as an epiphenomenon of lateral inhibition without containing any information that is actually used by the animal.

Research into the possible temporal processing of olfactory information has investigated two broad categories of odorant-linked temporal patterning of activity, namely: (1) oscillatory responses and (2) slow temporal patterning of responses. We will discuss each type of temporal patterning in the following section.

\section{Temporal patterning of olfactory responses}

\section{Oscillatory responses}

Odors induce periodic activity in the olfactory nervous systems of many species, including a mollusk (Limax maximus) [83], the locust form of the American bird grasshopper (Schistocerca americana) [84], a moth (Manduca sexta) [85], honeybees [86], zebrafish [87], and rodents [88]; it therefore has been implied that these oscillations are of fundamental importance to olfactory information processing $[15,89,90]$. However, it should be noted that oscillations are not observed in fruit flies [91] despite their robust olfactory behaviors [92, 93]. High-frequency oscillations also have not been observed in very young rats [94], which display odor-guided approach and avoidance behaviors [95, 96], as well as learning and memory of odorant discriminations that are similar to adults [96]. The presence of an entire repertoire of odor-guided behaviors in the absence of oscillations clearly indicates that these oscillations are not required for the behaviorally guided olfactory responses that have been studied. Finally, the first presentations of an odorant do not evoke oscillations in any of the species that have been studied. Rather, the odorants must be presented repeatedly in a certain rhythm to prime the oscillations [97, 98], and while most of the oscillation research entirely ignores the initial responses to any odorant, we are not aware of any behavioral data that would justify this omission of these data in an analysis of odor information processing. To the contrary, as we will discuss later in this review, behavioral responses to odorants occur rapidly after their initial presentation. Much of this work has been done with the locust, but there has been no demonstration that locusts actually ignore the first contact with odorants in favor of later stimuli, although it has been speculated that the repeated olfactory stimulation might not be perceived initially, but would somehow prepare the animals to respond to odorants reliably present in the environment [98]. At the same time, it seems highly unlikely that a flying insect that requires rapid responses to olfactory cues to be able to move up a patchy odorant plume would be insensitive to odorants during initial transient exposures to these critical cues.

The oscillations in local field potential that have been measured in the mushroom body of locusts are caused by the synchronized firing of antennal lobe projection neurons, whose projections terminate onto Kenyon cells in the mushroom body [84]. Upon odorant presentation, individual antennal lobe neurons contribute spikes to particular cycles of the oscillation, but reliably do not fire during other cycles [77]. Antennal lobe neurons also project to neurons of the lateral horn, causing oscillations in this adjacent structure in the protocerebrum [99]. The lateral horn neurons then relay out-of-phase inhibition onto the Kenyon cells, thereby effecting a temporal sharpening of the responses in these cells, resetting their responses every $50 \mathrm{~ms}$, and helping to insure that each Kenyon cell responds to a more limited set of odorants than does a typical antennal lobe projection neuron [99].

In both locusts and honeybees, the mushroom body oscillations can be blocked by the presence of the GABA receptor antagonist picrotoxin in the antennal lobe, showing the importance of inhibitory synapses between local interneurons and projection neurons in the antennal lobe in generating the oscillations [100, 101]. When picrotoxin was applied to the honeybee antennal lobe to block oscillations during conditioning of a proboscis extension response to the alcohol 1-hexanol, the treated bees later showed generalization by also responding to another alcohol, 1-octanol, a pattern of behavior that did not occur for untreated bees [101]. However, the picrotoxin-treated honeybees successfully discriminated 1-hexanol from geraniol, a longer, branched, double-bonded primary 
alcohol. Another study showed that picrotoxin also caused generalization from 1-hexanol to 1-octanol when applied during the retrieval phase of the same task [102], and the retrieval effect recently has been extended to the moth $M$. sexta using 2-octanone and 2-hexanone as similar odorants and either picrotoxin or bicuculline methyliodide as the GABA antagonist [103]. Since treated bees continued to detect and respond appropriately and differentially to the conditioned odorant, the data clearly eliminate oscillations as critical mechanisms for either odor detection or odor quality perception. In addition, because GABA receptor antagonists can reduce olfactory sharpening either by eliminating lateral inhibition or oscillations [58], their effect on discrimination cannot be conclusively attributed to either mechanism to the exclusion of the other. In fact, reduced lateral inhibition would likely increase the number of neurons activated by both 1-octanol and 1-hexanol, a finding that may be relevant to the observed generalization in the picrotoxin-treated bees. One possibility is that when conditioning occurred in the presence of picrotoxin in the antennal lobe, 1-hexanol could have stimulated a larger set of projection neurons than in untreated bees, including some of those neurons that would have responded to 1-octanol in untreated bees. The synapses involved in the representation of 1-octanol therefore would have been strengthened during the conditioning to 1-hexanol. When memory was later tested in the treated bees, 1-octanol would be expected to lead to a greater response than it did in untreated bees. Similarly, when picrotoxin was applied during retrieval, 1-octanol would be expected to stimulate a greater number of projection neurons than it would have in untreated bees, including some of those neurons that would have responded to 1-hexanol in untreated bees. Because the conditioning already would have strengthened the synapses involved in the 1-hexanol response, 1-octanol would be expected to give a greater response than in untreated bees. When moths were trained explicitly to discriminate odorant pairs, they could continue to distinguish closely related odorants at high concentrations even during GABA receptor blockade [90], a finding consistent with the oscillation-free model, given the expectation that only the differential responses to the two odorants would be strengthened during the initial training, and that these differential responses should be maintained during treatment.

Correlations between olfactory bulb or olfactory cortex oscillations and olfactory performance have been noted in rats [104-107], and these findings can be explained as relationships between oscillations and top-down mediation of olfactory system activity due to arousal, attention, frustration, or stress, rather than relationships between oscillations and odor quality. For example, olfactory bulb gamma oscillations increase over repeated odorant presentations after the learning of difficult, but not easy, two-odorant alternative choice tasks [104]. However, while the oscillations do not occur during the early trials of each testing session, the animals consistently perform accurately in these early trials, even for difficult discriminations, showing that the increased oscillations are not needed for the initial differential perception of the odors [104]. Transgenic mice lacking certain GABA receptors on granule cell inhibitory neurons in the bulb exhibited increased oscillations and also discriminated closely related odorants better than did wild-type controls [108]. However, the likely corresponding increase in lateral inhibition of mitral cells following this decreased inhibition of granule cells would be expected to limit the responsiveness of individual mitral cells to fewer odorants [28], a possibility that might underlie the enhanced odorant discrimination.

Electrical stimulation of the olfactory bulb can generate both gamma oscillations in olfactory cortex and behaviors related to odor perception, but higher levels of stimulation are required to achieve evidence of odor perception than are needed to elicit oscillations, thereby showing that the presence of evoked oscillations can be unlinked from odor quality perception [109]. These data suggest that olfactory system oscillations, or the temporal patterns of response in specific neurons that they may reflect, have not been shown to play a critical role in mediating initial olfactory perception.

\section{Slow temporal patterning of responses}

The temporal patterns of action potentials and periods of silence are reasonably reliable for the same mitral cell or antennal lobe projection neuron upon repeated presentations of the same odorant, and these temporal patterns differ for different odorants and different neurons [74, 77, 110-113]. Because of the reliable differences in temporal patterns evoked by different odorants, it has been proposed that odor quality information might be contained in these slow temporal patterns.

In locusts, the temporal patterns of projection neuron responses typically extend for several seconds, even when the odorant stimulus is presented only as a brief pulse, and although responses continue during the entire duration of a sustained 10-s odorant stimulus, more dramatic and odorant-specific changes occur during 2-s periods after the onset and the offset of the stimulus pulse [114]. In zebrafish, chemically similar amino acids evoke correlated temporal activity patterns across an ensemble of mitral cells early, but not later in the response, whereas more chemically dissimilar amino acids evoked correlated temporal patterns only later in the response, and it was suggested that the later time points could be used to discriminate between odorants that would be classified together on the basis of chemistry during the early portions 
of the response $[112,115]$. A separate consideration of late mitral cell responses that are synchronized with the local field potential, compared to late responses that are not synchronized, also was suggested as a means to classify and discriminate responses simultaneously [116].

Multivariate mathematical analyses involving ensembles of zebrafish mitral cells and locust projection neurons have suggested that the theoretical information content of these responses can be increased by considering changes in response over time [112, 114-119]. Classification tests also were used in these studies, wherein the response to an unknown odorant was represented as a time series of the activity of an ensemble of neurons and was compared to investigator-generated templates based on average recorded responses during other odorant presentations that also were comprised by time series of activity. For example, activity at $400 \mathrm{~ms}$ for the unknown odorant was compared to activity at $400 \mathrm{~ms}$ for a known odorant template, and the unknown odorant activity at 1,000 ms was compared to the known odorant template activity at 1,000 ms. Odorants could be correctly classified computationally with this temporally matched information, especially at later time points [112, 114-119].

Although such analyses have established the theoretical possibility of "information content" in slowly developing temporal activity patterns, there are important problems with accepting these models as a biological explanation for how odorant stimuli evoke perceptions of odor. We will present a list of these issues and then discuss each issue in detail. First, behavioral data are better predicted by a simpler analysis of the identity of activated neurons than by an analysis of temporal activity patterns. Second, behavioral data from several species show that animals can detect, discriminate, and respond to odorants before most of the described temporal patterns are established, suggesting that much or all of what we think of as odor coding cannot be explained by these slow processes. Third, the oscillatory mechanisms that have been investigated in the mushroom bodies of locusts allow odorant processing for only brief intervals, preventing any readout of an ongoing, slow temporal progression in the antennal lobe. Fourth, a mechanism for matching incoming odorant information with stored information about temporal activity patterns for numerous learned odorants has not been reported. Finally, different temporal patterns of electrical microstimulation are not discriminated under conditions where different spatial patterns are discriminated.

\section{Behavioral predictions}

To our knowledge, no behavioral study of odor perception has been published on the locust, the species on which much of this work has been done, although these insects appear to respond differentially to odorants [120]. The few specific perceptual predictions that have been based on the analysis of odorant-evoked temporal patterns in this species therefore remain untested. In zebrafish, responses to chemically related odorants are described as becoming decorrelated from each other late in the temporal progression of the activity patterns [112, 115], and yet behavioral tests show that zebrafish and catfish typically do not distinguish the odors of these amino acids, but instead respond in a manner predicted by the initial, overlapping glomerular activity patterns [8]. In fact, the mathematical analyses of electrophysiological data from zebrafish show new correlations involving responses to chemically dissimilar amino acids arising later in the temporal progression $[112,115]$, and yet there are no behavioral data showing that zebrafish show new perceptual generalizations with prolonged odorant sampling. Honeybees respond effectively in behavioral tasks involving the entirely natural presentation of odorants for sustained periods [101, 102, 121], an observation that calls into question the relevance of analyses that conclude that there is a greater difference between activity patterns during the dynamic transients occurring at odorant onset and offset [114]. On the other hand, the relative levels of stimulation of antennal lobe glomeruli accurately predict the patterns of generalization between odorants in honeybees [11].

\section{Temporal constraints on temporal coding}

In contrast to the relatively slow development of temporal activity patterns in the olfactory system over the course of several seconds, it is now clear that many animals can detect, process, and respond appropriately and differentially to odorants in a few hundred milliseconds. Any use of temporal information for these tasks must be limited to only a portion of the time taken by the entire task, given the time taken for odorant detection in the nose and the time for the motor component of the response. For example, mice can respond differentially to odorants within $\sim 200 \mathrm{~ms}$ when performing a well-learned, easy, go/no-go two-odorant discrimination task, although they choose to spend up to 300-500 ms for very difficult tasks involving discrimination between either binary mixtures containing the same components in similar ratios or two similar concentrations of the same odorant [122]. Rats also take about $200 \mathrm{~ms}$ to sample, and then about $300 \mathrm{~ms}$ to respond to odorants in a well-learned two-odorant alternative choice discrimination task, independently of the difficulty of the task [123]. Spontaneous sampling times of $\sim 260 \mathrm{~ms}$, independent of task difficulty, were confirmed in mice performing a two-odorant alternative choice, although artificially imposing longer sampling times up to $\sim 600 \mathrm{~ms}$ 
increased the accuracy of their performance [124]. The fact that mice do not choose to take full advantage of the longer sampling times on their own suggests that these experiments may be using a somewhat contrived set of conditions to demonstrate the value of increased processing time.

Furthermore, the increases in sampling time for difficult tasks and the increase in accuracy with time in these tasks may not even involve the progressions in temporal patterns of activity in the mitral cells. Cortical responses begin well before additional sampling improves performance [70], and cognitive processing of odorant information independent from ongoing activity in the olfactory bulb seems quite likely during that initial sampling period. Also, these timedependent tasks involve extremely fine discriminations of odorant mixtures (e.g., discriminating a 55:45 mixture from a 45:55 mixture) that are extraordinarily difficult to learn and perform, involving thousands of trials to establish [122-124]. Thus, the tasks may not reflect the kinds of olfactory information processing that these animals typically would use in the wild, for which the shorter processing times likely would be adequate. Indeed, spontaneous respiratory responses to a novel odorant following habituation to another odorant occur within $140 \mathrm{~ms}$ of inhaling the odorant, demonstrating that rapid olfactory processing occurs even for odorants that have not been learned [125].

A male housefly chasing a female can execute a corrective turn within $40 \mathrm{~ms}$ after a course deviation by its target [126], and this rotational stabilization reflex is directly activated by odor cues [127]. These data demonstrate the capacity for a remarkably brief minimum processing time for olfactory cues in an insect.

On the other hand, the gradual declustering of randomly recorded mitral cell responses in zebrafish reaches a maximum after $800 \mathrm{~ms}$ following odorant onset [112]. It is not known whether zebrafish are better able to sense or respond differentially to odorants after that time or whether their perceptions are in fact rapid and stable, with little involvement of prolonged responses. Locusts respond to some odorants with an oscillatory response only after about a second [84], and they have an oscillatory response to other odorants only upon a second presentation [97], a response delay that would suggest even slower perception if these oscillations were critical for odor perception.

Given the knowledge of fast olfactory perception, it seems particularly important to determine the minimum time needed for perception in both locusts and zebrafish to evaluate the utility of any slow temporal response pattern in the mechanisms underlying those perceptions. Clearly, neural responses that occur after the perception occurs can have no critical role in eliciting that perception.
Periodic re-setting of responses in secondary neurons

The antennal lobe projection neurons in insects that display the slow temporal activity patterns project to Kenyon cell secondary neurons in the mushroom bodies, as well as to neurons in the lateral horn. In the locust, Kenyon cells respond to far fewer odorants than do the antennal lobe projection neurons, and these responses are confined to only a fraction of the time involved in the slow temporal patterns due to a periodic inhibition relayed into the mushroom bodies every $50 \mathrm{~ms}$ by oscillating neurons in the lateral horn [99] and due to inhibition by mushroom body recurrent interneurons [128]. A similar narrowing of the temporal window for Kenyon cell responses has been found using calcium imaging in honeybees, where the responses occur within the first $200 \mathrm{~ms}$ and have entirely ceased by $600 \mathrm{~ms}$ [128]. Given this transformation of activity into brief phasic responses, there is no reason to believe that a mechanism exists by which information in the slow temporal patterns can be read by neurons at higher stages of processing.

Mechanism for playback of stored information?

In addition to the physiological evidence for regular interruptions during the read-in of slow odorant-evoked temporal activity patterns, there also are difficulties with the eventual read-out of information represented as a stored temporal pattern. Classification tests that have been used to buttress models of temporal coding of odor quality generally match an incoming temporal pattern of activity with a set of experimenter-generated template patterns on a time bin-by-time bin basis $[114,115,117-119]$. If such classification tests are meant to be biologically relevant, then each odorant presentation must be accompanied in the olfactory system by simultaneous, synchronized neural playbacks of stored information from memory about all possible corresponding odorants. However, no evidence has been provided for such a process in any olfactory system. Moreover, it is not clear how such information would be stored by neurons, how the patterns would be synchronized with the odorant stimulus and response, and where in the olfactory brain the comparisons would be made.

Microstimulation experiments do not reveal a temporal dimension

An elegant approach to determining the relative importance of neural identity or temporal representations of olfactory information involved microstimulation of the rat olfactory bulb using arrays of electrodes and different spatial or temporal patterns of stimuli. Rats began sniffing in the 
direction of the air flow when their bulbs were stimulated, consistent with their perceiving a novel odorant, and the electrical stimulus could replace an odorant stimulus in toxicosis conditioning $[129,130]$. The rats also could be taught to discriminate between electrical stimulation in different locations within the glomerular layer as if the different locations evoked the perception of different odors, and the difficulty of learning the discrimination was inversely correlated both with physical distance between the positively and negatively associated electrodes and with the degree of overlap of stimulated electrodes in the arrays [131]. These results demonstrate the importance of the identity of the stimulated neurons in olfactory perception without any difference in the temporal aspects of the stimulation. However, the rats did not learn to discriminate between the stimulation of the same sets of electrodes at different times during the respiration cycle [132]. The fact that rats could not discriminate between clear differences in temporal patterns of stimulation argues against the importance of a temporal code for olfaction as it is presently conceived.

An alternative explanation for the presence of the slow temporal patterns is that they are background responses that are recorded from randomly selected neurons, whereas other neurons that are highly activated elsewhere in the system rapidly carry the coded information. Background responses would be expected to vary in response time as a function of their distance from the highly activated neurons, thereby giving the impression of carrying temporal information regarding the odorant cues.

\section{Ensemble coding of olfactory information?}

The slow temporal patterns of activity evoked by odorants in mitral cells and projection neurons change greatly with the concentration of a single odorant in many species [75, $76,110,111,117]$. The perception of odor quality is generally found to be consistent across odorant concentration, leading to the conclusion that temporal patterns in individual cells should not be able to code odor quality by themselves [111]. In rats, the transformation of the chemosensory input into relative levels of activity by the olfactory bulb appears to be critical to allow for concentration-invariant perception of most odors, and we have suggested that short-axon cells in the glomerular layer transform glomerular input into a normalized response by suppressing mitral cell activity throughout the bulb to an extent reflecting the average level of glomerular input [133]. Indeed, the presence of relative differences in glomerular response may be critical for the olfactory bulb to carry any information about odor. When about $95 \%$ of the olfactory sensory neurons of mice were made to express the same odorant receptor, a ligand for that receptor evoked strong responses across the entire bulb, but the animals showed no evidence of perceiving the odor without a differential glomerular response [134].

When an ensemble of about 100 projection neurons in locusts was analyzed, temporal progressions allowed intensity-independent classifications of odor quality, presumably because different neurons displayed their intensity-dependent changes at different odorant concentrations. In this experiment, individual 50-ms time bins were able to classify odorants correctly with respect to quality independently of intensity, and peak success occurred very quickly $(<300 \mathrm{~ms})$, suggesting that virtually all of the temporal progression was unnecessary for effective classification [117]. In other words, when slow temporal dynamics were essentially eliminated, the experimenter could still accurately predict the odorant concentration from the differential response of a large number of neurons. The data therefore lend support to the idea of an identity code rather than a temporal code for odor concentration, since the olfactory information was critically dependent on the activation of specific neurons, rather than slowly evolving temporal responses.

Even without considering temporal patterns, different researchers might disagree on how many receptors, glomeruli, and projection neurons are involved in the perception of each individual odorant. Nearly all researchers agree that most olfactory stimuli, including pure odorant chemicals, are represented by activity originating in multiple receptor types, but their notions range from a combinatorial code involving a limited number of types to a more highly distributed representation involving nearly all neurons equally at each level of olfactory processing. In the experiment regarding odorant intensity in the locust, subsets of as few as ten projection neurons could be used to get $>75 \%$ accuracy of classification, provided that the "right" ten neurons were considered [117]. The sets of ten neurons that were identified as potentially bearing correct information in that study were selected randomly, but it would be interesting to consider whether those neurons would turn out to be the ones most directly associated with the most activated glomeruli. The data used to support a notion of a temporal code so far seems instead to offer strong support for an identity code for odor quality.

Receptors necessarily respond similarly to molecularly similar odorants due to the electrical and steric interactions between odorant ligands and protein binding sites. It has been suggested that the function of the antennal lobe or olfactory bulb is to decorrelate responses so that even very similar odorants can be given the most distinct central representations [14]. The circuitry of the antennal lobe or olfactory bulb has been proposed to accomplish that task by distributing differential activity across numerous 
projection neurons so that the entire ensemble has a temporal activity pattern that maximally distinguishes all odorants from each other, even those odorants that resemble one another chemically [14, 113, 115, 116, 135]. The activity of particular neurons in this ensemble is not considered to be important to the new representation. Instead, activity across the projection neuron ensemble is thought to create a new "representational space" in which each "odor" is nearly equally distant from all other odors, as opposed to the "stimulus space" that is constituted by the odorant chemicals themselves, where similarities in structure create local response clusters that would be associated with poorer perceptual discrimination [14]. In this view, the now re-coded information, which should be less correlated with odorant chemistry, then is passed on to Kenyon cells, which are the targets in the mushroom body of the antennal lobe projection neurons [14]. The Kenyon cells are proposed to generate a "sparsened" version of this code wherein fewer odorants stimulate each cell than was the case for the projection neurons. Again, it is proposed that the differences in the signals evoked between all odorants in the mushroom body should be equally maximized.

If this scenario were true, then Kenyon cells should be much less activated by related odorant chemicals than are sensory neurons. However, the published examples of activity in locust Kenyon cells or their targets across a panel of related odorant chemicals show apparent evidence of similar responses to closely related odorant chemicals, just as would be observed for receptors or glomeruli of the rat olfactory bulb. In one report, a Kenyon cell responded best to 1-octanol, with overlapping responses to 1-heptanol, a closely related alcohol, and with slightly lesser responses to other related alcohols, 1-hexanol and 1-cis-3-hexen-1-ol [99]. The cell also responded to aldehydes such as octanal and nonanal of similar chain length to the alcohols, but not to various ketones, mint, cherry, or a nitrile. This response profile is quite similar to that seen in the vertebrate olfactory bulb, where responses also are clearly related to odorant chemistry [6, 136-140]. Indeed, even locust betalobe neurons, which receive input from the Kenyon cells, show evidence of the same type of overlapping responses to similar odorants (pentanol $>$ hexanol $>$ octanol) [141]) that are seen in rodent receptors and glomeruli [136-140]. Very similar observations can be made regarding responses of Kenyon cells in fruit flies, which respond to pairs of ethyl esters that are neighbors in homologous series or to aliphatic alcohols and aldehydes of similar carbon number [142], response profiles quite similar to those of individual odorant receptors recorded from specific sensory neurons in fruit fly antennae [143].

The molecular receptive ranges of these insect mushroom body neurons therefore suggest that responses have not been so much decorrelated as they have been filtered, isolating the olfactory signal from the noise in the antennal lobe activity, and relaying to the Kenyon cells a sparse, probably tuned activity profile that appears to reflect specifically activated receptors and glomeruli surprisingly well. In other words, the circuitry may identify a smaller subset of projection neurons that efficiently represent the odorant with a greatly elevated response above the lowlevel background responses that may be recorded in randomly selected neurons during odorant presentations. It is our hope that observations such as these might motivate a formal study of the relationships between stimulus and response at various levels in a variety of organisms, especially between sensory neurons and Kenyon cells. Ultimately, we think it will be necessary to characterize which projection neuron responses are actually responsible for the Kenyon cell activity. The mathematical evidence for sparsening between antennal lobe neurons and Kenyon cells may simply indicate that too many antennal lobe neurons were being included in the analyses, given both the arbitrarily located recordings of neurons at that stage and the liberal definition of a response.

Systematic studies of stimulus and response in which neurons have not been selected randomly for recording have recently been conducted in fruit flies at the olfactory sensory neuron and antennal lobe levels. When postsynaptic activity in projection neurons was monitored using optical imaging of $\mathrm{Ca}^{2+}$ signals, activity profiles were found to be very similar to those observed upon imaging presynaptic glomerular responses $[9,59]$. However, when responses of projection neurons were recorded using electrophysiology and were broadly defined to include late changes in activity, responses were concluded to be unrelated to glomerular activity, although weak correlations arose when only the first $100 \mathrm{~ms}$ of the response was considered [113]. The difference in result from that of the optical imaging studies was attributed to dissociation between postsynaptic $\mathrm{Ca}^{2+}$ signals and action potentials [113]. However, in a later electrophysiological study involving more glomeruli, a median correlation coefficient ( $r$ ) of 0.84 was found to describe the relationship between projection neuron and glomerular activity [63]. These data seem to us to be an impressively high correlation that provides support for an identity code, despite the rare mismatches in activity between glomeruli and projection neurons. It should be noted that the correlation coefficients revealed in such studies depend in part on the choice of odorants - a different result would be obtained if one of the relevant receptors responds to groups of related odorants in the chosen panel than if the receptors only respond to a few of the odorants. If the most effective odorants are omitted from the panel, then the results may not be particularly meaningful. One also should distinguish between a truly 
broad responsiveness and what may be better thought of as narrow tuning to more than one class of odorant, which in turn might suggest interesting experiments about special interactions between particular odorants. In any case, it seems apparent that the more odorants that are studied across many identified neurons at different levels in the system, the better our understanding of the specificity of the neurons to olfactory cues.

As a more direct test of the importance of individual receptors and glomeruli to the perception of odors in fruit flies, innate preferences for the pheromone cis-vaccenyl acetate and the nonpheromonal odorant geranyl acetate were tested in flies with genetic ablations of receptors that were narrowly tuned to each of these odorants [144]. In both cases, the spontaneous behavioral responses to the odorants were blocked by ablating just the corresponding single receptor, revealing a critical involvement of individual receptors in these odor-guided responses [144]. An essential involvement of a single receptor certainly would not be consistent with a highly distributed code wherein all neurons are equally important. Rather, a simple identity code would be supported by such data, despite previous observations of other receptors that are activated by geranyl acetate and despite responses by projection neurons associated with the deleted receptor's glomerulus to odorant chemicals unrelated to geranyl acetate. Speculation regarding a broadly responsive ensemble representation depending on temporal information rather than the identity of the activated neurons does not seem necessary to explain the results.

Research on other simple behavioral responses in other model species also has supported a special importance of individual receptors or sensory neurons as tested by targeted ablation. The $\mathrm{V}$ glomerulus is vital to carbon dioxide avoidance in fruit flies [145], the odr-10 receptor gene has special involvement in diacetyl-guided chemotaxis in the roundworm (Caenorhabditis elegans) [146], and a single sensory neuron is important for chemotactic responses to several other odorants in C. elegans [147]. These results strongly support an identity code in which particular neurons carry the information about the odorants that are involved in the specific behaviors.

In rodents, it is clear that multiple receptors and glomeruli are involved in the response to most odorant chemicals. For one thing, there are groups of distinct receptor genes that code for receptor proteins of related specificity [137], and sensory neurons expressing these related receptors project to neighboring glomeruli in the olfactory bulb to form response domains or modules that respond to related odorants [2, 3]. Many odorants have several distinct molecular features that bind to distinct sets of receptors; a single odorant can activate one group of receptors whose specificity is related to the odorant's functional group as well as activating a distinct group of receptors whose specificity is related to the odorant's hydrocarbon structure [3, 136-140]. Because the corresponding glomeruli are contained within distinct domains of the olfactory bulb, the activity can occur as an ensemble involving a few clusters of glomeruli.

Kobayakawa et al. [148] determined how the loss of specific glomeruli in the mouse could affect the perception of specific odors. They developed mutant mice in which specific olfactory sensory neurons that project to the dorsal aspect of the olfactory bulb were eliminated by targeted expression of the diphtheria toxin gene. They found that these mice did not display their normal innate responses to the aversive odorants that normally evoke responses in that area [148]. These data constitute additional strong and direct support for the notion of an identity code.

The scattered distribution of areas of focal activation can explain how odor detection and discrimination between other odorants can be remarkably resistant to physical ablation of large parts of the bulb [149, 150]. The bulbar ablation studies have not shown the result predicted by a highly distributed ensemble code - the lesions do not cause a gradual loss of olfactory abilities that is proportional to the amount of bulb removed. In fact, the remaining abilities actually suggest a very special involvement of the spared areas. Indeed, the spared areas invariably contained sufficient focal responses to allow well-trained rats to make olfactory discriminations between the odorants. It is also relevant that the apparatus used to determine the efficacy of olfactory function in these animals may allow discriminations to occur without the use of olfactory cues (see reference [3] for a detailed discussion of these data). Indeed, mice in which $95 \%$ of olfactory sensory neurons express a single gene had largely normal olfactory responses when tested in this apparatus, while they appeared to be virtually anosmic when tested in two other behavioral paradigms [134]. It is also the case that rats can perform discriminations in this apparatus after their olfactory bulbs are completely removed [151].

In a related test of identity coding, fruit flies expressing only a single functional Or83b-coupled receptor type in their olfactory sensory neurons were found to be capable of discriminating pairs of odorants that stimulate that receptor [152]. This receptor was not stimulated by all odorants, so that fruit flies require more than one receptor to detect and discriminate a full range of odorants. Nevertheless, these data present obvious questions for both an identity code and a temporal code. Intact flies may normally use an identity code to identify and discriminate all odors, given that an individual odorant commonly stimulates more than one receptor type, and yet the flies may be able to compensate for the loss of the normal information by using alternative, temporal cues. The proposal that these flies use temporal information would be strengthened considerably 
through a demonstration that the single odorant receptor in fact generated differential temporal responses for different odorants. It also will be important to determine if Or83bindependent families of olfactory receptors in flies might contribute to discrimination (as opposed to detection) of the tested odorants [153].

\section{Temporal issues in other coding scenarios}

The timing of signals in the olfactory system is probably of great importance, even if temporal patterns themselves do not carry information about odor quality. Once attention turns to a characterization of how odorants affect actual animal behaviors, we can hope to gain a better understanding of the significance of response timing. Potential factors include temporal "binding" and coincidence detection to enhance signals over noise and to unite responses both to different components of mixtures and to distinct features of individual odorants [154, 155]. Conveying the strength of a signal from one level of processing to another, whether as a firing rate (action potentials arriving within a temporally summed window), or as a delay between action potentials and some reference time such as a respiratory rhythm [156], seems by definition to involve a temporal code. Indeed, a relative latency-based coding system in the retina for detecting a visual signal at various levels of contrast has recently been shown to have advantages over coding schemes based on rates of action potentials [157]. Another particularly convincing example of how response timing can convey specialized information in olfaction is the determination of odorant location by using time differences in the arrival of odorant stimuli at each rat naris [158].

\section{Summary}

The critical importance of specific neurons in the process of olfactory perception has been demonstrated repeatedly in the data that have been published to this point. Elimination of even a single receptor can suppress olfactoryguided behavior toward a specific odorant, and conversely, electrical microstimulation of specific neurons in the system can evoke different olfactory-guided behaviors. On the other hand, the dominant notions of temporal coding have not been well supported by the available data. To advance this field, we suggest that proposals regarding olfactory coding be tested using perceptually driven behavior, rather than information optimization as its starting point. Furthermore, any notion of temporal coding should also be constrained by the minimum time used by the system to support olfactory perception, and this parameter should be characterized in each olfactory system that is being studied. In addition, one should be able to impose on the system what is thought to be the temporal signal using microstimulation and thereby evoke an olfactory-guided behavior. Conversely, suppression of what is thought to be the critical temporal signal without the suppression of other neural processes that are involved in olfactory processing should suppress olfactory-guided behavior. When such experiments are completed for current notions of temporal coding, as they have been for current notions of identity coding, we will be better able to evaluate the importance of any temporal aspect of the processed signal in the olfactory system of a particular species in the production of a particular olfactory behavior. As it stands, however, much of the data that purports to support temporal coding in olfaction either is inconsistent with those hypotheses or actually supports an identity code for olfaction.

Acknowledgments Our work has been supported by United States Public Health Service Grants DC03545, DC006391, and DC006516. We are grateful to an anonymous reviewer for many excellent suggestions following a very careful reading of our manuscript.

Open Access This article is distributed under the terms of the Creative Commons Attribution Noncommercial License which permits any noncommercial use, distribution, and reproduction in any medium, provided the original author(s) and source are credited.

\section{References}

1. Adrian ED (1954) The basis of sensation: some recent studies of olfaction. Br Med J 4857:287-290

2. Mori K, Takahashi YK, Igarashi KM, Yamaguchi M (2006) Maps of odorant molecular features in the mammalian olfactory bulb. Physiol Rev 86:409-433

3. Johnson BA, Leon M (2007) Chemotopic odorant coding in a mammalian olfactory system. J Comp Neurol 503:1-34

4. Youngentob SL, Johnson BA, Leon M, Sheehe PR, Kent PF (2006) Predicting odorant quality perceptions from multidimensional scaling of olfactory bulb glomerular activity patterns. Behav Neurosci 120:1337-1345

5. Friedrich RW, Korsching SI (1997) Combinatorial and chemotopic odorant coding in the zebrafish olfactory bulb visualized by optical imaging. Neuron 18:737-752

6. Friedrich RW, Korsching SI (1998) Chemotopic, combinatorial, and noncombinatorial odorant representations in the olfactory bulb revealed using a voltage-sensitive axon tracer. J Neurosci 18:9977-9988

7. Nikonov AA, Caprio J (2005) Processing of odor information in the olfactory bulb and cerebral lobes. Chem Senses 30(Suppl 1): $\mathrm{i} 317-\mathrm{i} 318$

8. Valentincic T, Miklavc P, Dolenšek J, Pliberšek K (2005) Correlations between olfactory discrimination, olfactory receptor neuron responses and chemotopy of amino acids in fishes. Chem Senses 30:i312-i314

9. Wang JW, Wong AM, Flores J, Vosshall LB, Axel R (2003) Two-photon calcium imaging reveals an odor-evoked map of activity in the fly brain. Cell 112:271-282 
10. Galizia CG, Sachse S, Rappert A, Menzel R (1999) The glomerular code for odor representation is species specific in the honeybee Apis mellifera. Nat Neurosci 2:473-478

11. Guerrieri F, Schubert M, Sandoz J-C, Giurfa M (2005) Perceptual and neural olfactory similarity in honeybees. PLoS Biol 3:e60

12. Laurent G (1997) Olfactory processing: maps, time and codes. Curr Opin Neurobiol 7:547-553

13. Laurent G (1999) A systems perspective on early olfactory coding. Science 286:723-728

14. Laurent G (2002) Olfactory network dynamics and the coding of multidimensional signals. Nat Rev Neurosci 3:884-895

15. Laurent G, Stopfer M, Friedrich RW, Rabinovich MI, Volkovskii A, Abarbanel HD (2001) Odor encoding as an active, dynamical process: experiments, computation, and theory. Annu Rev Neurosci 24:263-297

16. Miyamichi K, Serizawa S, Kimura HM, Sakano H (2005) Continuous and overlapping expression domains of odorant receptor genes in the olfactory epithelium determine the dorsal/ ventral positioning of glomeruli in the olfactory bulb. J Neurosci 25:3586-3592

17. Serizawa S, Miyamichi K, Sakano H (2004) One neuron-one receptor rule in the mouse olfactory system. Trends Genet 20:648-653

18. Zhang X, Rodriguez I, Mombaerts P, Firestein S (2004) Odorant and vomeronasal receptor genes in two mouse genome assemblies. Genomics 83:802-811

19. Ressler KJ, Sullivan SL, Buck LB (1994) Information coding in the olfactory system: evidence for a stereotyped and highly organized epitope map in the olfactory bulb. Cell 79:1245-1255

20. Vassar R, Chao SK, Sitcheran R, Nuñez JM, Vosshall LB, Axel $R$ (1994) Topographic organization of sensory projections to the olfactory bulb. Cell 79:981-991

21. Treloar HB, Feinstein P, Mombaerts P, Greer CA (2002) Specificity of glomerular targeting by olfactory sensory axons. J Neurosci 22:2469-2477

22. Sharp FR, Kauer JS, Shepherd GM (1975) Local sites of activity-related glucose metabolism in rat olfactory bulb during olfactory stimulation. Brain Res 98:596-600

23. Johnson BA, Woo CC, Hingco EE, Pham KL, Leon M (1999) Multidimensional chemotopic responses to $n$-aliphatic acid odorants in the rat olfactory bulb. J Comp Neurol 409:529-548

24. Johnson BA, Leon M (2000) Odorant molecular length: one aspect of the olfactory code. J Comp Neurol 426:330-338

25. Johnson BA, Farahbod H, Xu Z, Saber S, Leon M (2004) Local and global chemotopic organization: general features of the glomerular representations of aliphatic odorants differing in carbon number. J Comp Neurol 480:234-249

26. Shepherd GM (1972) Synaptic organization of the mammalian olfactory bulb. Physiol Rev 52:864-917

27. Wachowiak M, Shipley MT (2006) Coding and synaptic processing of sensory information in the glomerular layer of the olfactory bulb. Semin Cell Dev Biol 17:411-423

28. Yokoi M, Mori K, Nakanishi S (1995) Refinement of odor molecule tuning by dendrodendritic synaptic inhibition in the olfactory bulb. Proc Natl Acad Sci USA 92:3371-3375

29. Ho SL, Johnson BA, Leon M (2006) Long hydrocarbon chains serve as unique molecular features recognized by ventral glomeruli of the rat olfactory bulb. J Comp Neurol 498:16-30

30. Ho SL, Johnson BA, Chen AL, Leon M (2006) Differential responses to branched and unsaturated aliphatic hydrocarbons in the rat olfactory system. J Comp Neurol 499:519-532

31. Linster C, Johnson BA, Morse A, Yue E, Xu Z, Hingco EE, Choi Y, Choi M, Messiha A, Leon M (2001) Perceptual correlates of neural representations evoked by odorant enantiomers. J Neurosci 21:9837-9843
32. Cleland TA, Morse A, Yue EL, Linster C (2002) Behavioral models of odor similarity. Behav Neurosci 116:222-231

33. Linster C, Johnson BA, Morse A, Yue E, Leon M (2002) Spontaneous versus reinforced olfactory discriminations. J Neurosci 22:6842-6845

34. Zhang X, Firestein S (2007) Comparative genomics of odorant and pheromone receptor genes in rodents. Genomics 89:441450

35. Robertson HM, Wanner KW (2006) The chemoreceptor superfamily in the honey bee, Apis mellifera: expansion of the odorant, but not gustatory, receptor family. Genome Res 16:1395-1403

36. Alioto TS, Ngai J (2005) The odorant receptor repertoire of teleost fish. BMC Genomics 6:173

37. Robertson HM, Warr CG, Carlson JR (2003) Molecular evolution of the insect chemoreceptor gene superfamily in Drosophila melanogaster. Proc Natl Acad Sci USA 100:14537-14542

38. Vosshall LB, Wong AM, Axel R (2000) An olfactory sensory map in the fly brain. Cell 102:147-159

39. Nikonov AA, Caprio J (2001) Electrophysiological evidence for a chemotopy of biologically relevant odors in the olfactory bulb of the channel catfish. J Neurophysiol 86:1869-1876

40. Sachse S, Rappert A, Galizia CG (1999) The spatial representation of chemical structures in the antennal lobe of honeybees: steps towards the olfactory code. Eur J Neurosci 11:3970-3982

41. Linster C, Sachse S, Galizia CG (2005) Computational modeling suggests that response properties rather than spatial position determine connectivity between olfactory glomeruli. J Neurophysiol 93:3410-3417

42. Lei H, Christensen TA, Hildebrand JG (2004) Spatial and temporal organization of ensemble representations for different odor classes in the moth antennal lobe. J Neurosci 24:1110811119

43. Nikonov AA, Finger TE, Caprio J (2005) Beyond the olfactory bulb: an odotopic map in the forebrain. Proc Natl Acad Sci USA 102:18688-18693

44. Nikonov AA, Caprio J (2004) Odorant specificity of single olfactory bulb neurons to amino acids in the channel catfish. J Neurophysiol 92:123-134

45. Nikonov AA, Caprio J (2007) Responses of olfactory forebrain units to amino acids in the channel catfish. J Neurophysiol 97:2490-2498

46. Nikonov AA, Caprio J (2007) Highly specific olfactory receptor neurons for types of amino acids in the channel catfish. J Neurophysiol 98:1909-1918

47. Derby CD (2000) Learning from spiny lobsters about chemosensory coding of mixtures. Physiol Behav 69:203-209

48. Kang J, Caprio J (1997) In vivo responses of single olfactory receptor neurons of channel catfish to binary mixtures of amino acids. J Neurophysiol 77:1-8

49. Deisig N, Giurfa M, Lachnit H, Sandoz JC (2006) Neural representation of olfactory mixtures in the honeybee antennal lobe. Eur J Neurosci 24:1161-1174

50. Silbering AF, Galizia CG (2007) Processing of odor mixtures in the Drosophila antennal lobe reveals both global inhibition and glomerulus-specific interactions. J Neurosci 27:1196611977

51. Belluscio L, Katz LC (2001) Symmetry, stereotypy, and topography of odorant representations in mouse olfactory bulbs. J Neurosci 21:2113-2122

52. Lin da Y, Shea SD, Katz LC (2006) Representation of natural stimuli in the rodent main olfactory bulb. Neuron 50:937-949

53. Grossman KJ, Mallik AK, Ross J, Kay LM, Issa NP (2008) Glomerular activation patterns and the perception of odor mixtures. Eur J Neurosci 27:2676-2685 
54. Bell GA, Laing DG, Panhuber H (1987) Odour mixture suppression: evidence for a peripheral mechanism in human and rat. Brain Res 426:8-18

55. Johnson BA, Ong J, Frederick D, Kay LM, Leon M (2008) Increased sniffing is associated with a behaviorally relevant suppression of dorsal glomerular responses observed for a binary mixture of unrelated odorants. ISOT XV Abstracts, International Symposium on Olfaction and Taste, 21-26 July 2008, p 439

56. Sharp FR, Kauer JS, Shepherd GM (1977) Laminar analysis of 2-deoxyglucose uptake in olfactory bulb and olfactory cortex of rabbit and rat. J Neurophysiol 40:800-813

57. Guthrie KM, Anderson AJ, Leon M, Gall C (1993) Odorinduced increases in c-fos mRNA expression reveal an anatomical "unit" for odor processing in olfactory bulb. Proc Natl Acad Sci USA 90:3329-3333

58. Sachse S, Galizia CG (2002) Role of inhibition for temporal and spatial odor representation in olfactory output neurons: a calcium imaging study. J Neurophysiol 87:1106-1117

59. Ng M, Roorda RD, Lima SQ, Zemelman BV, Morcillo P, Miesenböck G (2003) Transmission of olfactory information between three populations of neurons in the antennal lobe of the fly. Neuron 36:463-474

60. Imamura K, Mataga N, Mori K (1992) Coding of odor molecules by mitral/tufted cells in rabbit olfactory bulb. I. Aliphatic compounds. J Neurophysiol 68:1986-2002

61. Mori K, Mataga N, Imamura K (1992) Differential specificities of single mitral cells in rabbit olfactory bulb for a homologous series of fatty acid odor molecules. J Neurophysiol 67:786-789

62. Luo M, Katz LC (2001) Response correlation maps of neurons in the mammalian olfactory bulb. Neuron 32:1165-1179

63. Bhandawat V, Olsen SR, Gouwens NW, Schlief ML, Wilson RI (2007) Sensory processing in the Drosophila antennal lobe increases reliability and separability of ensemble odor representations. Nat Neurosci 10:1474-1482

64. Haberly LB (2001) Parallel-distributed processing in olfactory cortex: new insights from morphological and physiological analysis of neuronal circuitry. Chem Senses 26:551-576

65. Haberly LB, Price JL (1977) The axonal projection patterns of the mitral and tufted cells of the olfactory bulb in the rat. Brain Res 129:152-157

66. Scott JW, McBride RL, Schneider SP (1980) The organization of projections from the olfactory bulb to the piriform cortex and olfactory tubercle in the rat. J Comp Neurol 194:519-534

67. Buonviso N, Revial MF, Jourdan F (1991) The projections of mitral cells from small local regions of the olfactory bulb: an anterograde tracing study using PHA-L (Phaseolus vulgaris leucoagglutinin). Eur J Neurosci 3:493-500

68. Illig KR, Haberly LB (2003) Odor-evoked activity is spatially distributed in piriform cortex. J Comp Neurol 457:361-373

69. Zou Z, Li F, Buck LB (2005) Odor maps in the olfactory cortex. Proc Natl Acad Sci USA 102:7724-7729

70. Wilson DA (2000) Comparison of odor receptive field plasticity in the rat olfactory bulb and anterior piriform cortex. J Neurophysiol 84:3036-3042

71. Wilson DA (2001) Receptive fields in the rat piriform cortex. Chem Senses 26:577-584

72. Wilson DA (2000) Odor specificity of habituation in the rat anterior piriform cortex. J Neurophysiol 83:139-145

73. Kauer JS (1974) Response patterns of amphibian olfactory bulb neurones to odour stimulation. J Physiol 243:695-715

74. Chaput M, Holley A (1980) Single unit responses of olfactory bulb neurones to odour presentation in awake rabbits. J Physiol 76:551-558
75. Meredith M (1986) Patterned response to odor in mammalian olfactory bulb: the influence of intensity. J Neurophysiol 56:572-597

76. Harrison TA, Scott JW (1986) Olfactory bulb responses to odor stimulation: analysis of response pattern and intensity relationships. J Neurophysiol 56:1571-1589

77. Laurent G, Wehr M, Davidowitz H (1996) Temporal representations of odors in an olfactory network. J Neurosci 16:3837-3847

78. Spors H, Grinvald A (2002) Spatio-temporal dynamics of odor representations in the mammalian olfactory bulb. Neuron 34:301-315

79. Spors H, Wachowiak M, Cohen LB, Friedrich RW (2006) Temporal dynamics and latency patterns of receptor neuron input to the olfactory bulb. J Neurosci 26:1247-1259

80. Wehr M, Laurent G (1999) Relationship between afferent and central temporal patterns in the locust olfactory system. J Neurosci 19:381-390

81. Christie JM, Westbrook GL (2006) Lateral excitation within the olfactory bulb. J Neurosci 26:2269-2277

82. Meredith M (1992) Neural circuit computation: complex patterns in the olfactory bulb. Brain Res Bull 29:111-117

83. Gervais R, Kleinfeld D, Delaney KR, Gelperin A (1996) Central and reflex neuronal responses elicited by odor in a terrestrial mollusk. J Neurophysiol 76:1327-1339

84. Laurent G, Davidowitz H (1994) Encoding of olfactory information with oscillating neural assemblies. Science 265:1872-1875

85. Heinbockel T, Kloppenburg P, Hildebrand JG (1998) Pheromone-evoked potentials and oscillations in the antennal lobes of the sphinx moth Manduca sexta. J Comp Physiol A 182:703-714

86. Okada K, Kanzaki R (2001) Localization of odor-induced oscillations in the bumblebee antennal lobe. Neurosci Lett 316:133-136

87. Tabor R, Yaksi E, Friedrich RW (2008) Multiple functions of GABA A and GABA B receptors during pattern processing in the zebrafish olfactory bulb. Eur J Neurosci 28:117-127

88. Bressler SL, Freeman WJ (1980) Frequency analysis of olfactory system EEG in cat, rabbit, and rat. Electroencephalogr Clin Neurophysiol 50:19-24

89. Gelperin A (2006) Olfactory computations and network oscillations. J Neurosci 26:1663-1668

90. Lledo PM, Lagier S (2006) Adjusting neurophysiological computations in the adult olfactory bulb. Semin Cell Dev Biol $17: 443-453$

91. Wilson RI, Laurent G (2005) Role of GABAergic inhibition in shaping odor-evoked spatiotemporal patterns in the Drosophila antennal lobe. J Neurosci 25:9069-9079

92. Davis RL (2005) Olfactory memory formation in Drosophila: from molecular to systems neuroscience. Annu Rev Neurosci 28:275-302

93. Chabaud MA, Devaud JM, Pham-Delègue MH, Preat T, Kaiser L (2006) Olfactory conditioning of proboscis activity in Drosophila melanogaster. J Comp Physiol A Neuroethol Sens Neural Behav Physiol 192:1335-1348

94. Fletcher ML, Smith AM, Best AR, Wilson DA (2005) Highfrequency oscillations are not necessary for simple olfactory discriminations in young rats. J Neurosci 25:792-798

95. Leon M (1987) Plasticity of olfactory output circuits related to early olfactory learning. Trends Neurosci 10:434-438

96. Wilson DA, Sullivan RM (1994) Neurobiology of associative learning in the neonate: early olfactory learning. Behav Neural Biol 61:1-18

97. Laurent G, Naraghi M (1994) Odorant-induced oscillations in the mushroom bodies of the locust. J Neurosci 14:2993-3004

98. Stopfer M, Laurent G (1999) Short-term memory in olfactory network dynamics. Nature 402:664-668 
99. Perez-Orive J, Mazor O, Turner GC, Cassenaer S, Wilson RI, Laurent G (2002) Oscillations and sparsening of odor representations in the mushroom body. Science 297:359-365

100. MacLeod K, Laurent G (1996) Distinct mechanisms for synchronization and temporal patterning of odor-encoding neural assemblies. Science 274:976-979

101. Stopfer M, Bhagavan S, Smith BH, Laurent G (1997) Impaired odour discrimination on desynchronization of odour-encoding neural assemblies. Nature 390:70-74

102. Hosler JS, Buxton KL, Smith BH (2000) Impairment of olfactory discrimination by blockade of GABA and nitric oxide activity in the honey bee antennal lobes. Behav Neurosci 114:514-525

103. Mwilaria EK, Ghatak C, Daly KC (2008) Disruption of GABAA in the insect antennal lobe generally increases odor detection and discrimination thresholds. Chem Senses 33:267-281

104. Beshel J, Kopell N, Kay LM (2007) Olfactory bulb gamma oscillations are enhanced with task demands. J Neurosci 27:8358-8365

105. Freeman WJ, Skarda CA (1985) Spatial EEG patterns, nonlinear dynamics and perception: the neo-Sherringtonian view. Brain Res Rev 10:147-175

106. Ravel N, Chabaud P, Martin C, Gaveau V, Hugues E, TallonBaudry C, Bertrand O, Gervais R (2003) Olfactory learning modifies the expression of odour-induced oscillatory responses in the gamma $(60-90 \mathrm{~Hz})$ and beta $(15-40 \mathrm{~Hz})$ bands in the rat olfactory bulb. Eur J Neurosci 17:350-358

107. Kay LM (2003) Two species of gamma oscillations in the olfactory bulb: dependence on behavioral state and synaptic interactions. J Integr Neurosci 2:31-44

108. Nusser Z, Kay LM, Laurent G, Homanics GE, Mody I (2001) Disruption of GABA(A) receptors on GABAergic interneurons leads to increased oscillatory power in the olfactory bulb network. J Neurophysiol 86:2823-2833

109. Jirsa R, Radil T (1997) Gamma activity in the piriform cortex and behavioral thresholds for electrical stimulation in the olfactory bulb. Acta Neurobiol Exp 57:11-20

110. Meredith M, Moulton DG (1978) Patterned response to odor in single neurones of goldfish olfactory bulb: influence of odor quality and other stimulus parameters. J Gen Physiol 71:615643

111. Wellis DP, Scott JW, Harrison TA (1989) Discrimination among odorants by single neurons of the rat olfactory bulb. J Neurophysiol 61:1161-1177

112. Friedrich RW, Laurent G (2001) Dynamic optimization of odor representations by slow temporal patterning of mitral cell activity. Science 291:889-894

113. Wilson RI, Turner GC, Laurent G (2004) Transformation of olfactory representations in the Drosophila antennal lobe. Science 303:366-370

114. Mazor O, Laurent G (2005) Transient dynamics versus fixed points in odor representations by locust antennal lobe projection neurons. Neuron 48:661-673

115. Friedrich RW, Laurent G (2004) Dynamics of olfactory bulb input and output activity during odor stimulation in zebrafish. $\mathrm{J}$ Neurophysiol 91:2658-2669

116. Friedrich RW, Habermann CJ, Laurent G (2004) Multiplexing using synchrony in the zebrafish olfactory bulb. Nat Neurosci 7:862-871

117. Stopfer M, Jayaraman V, Laurent G (2003) Intensity versus identity coding in an olfactory system. Neuron 39:991-1004

118. Broome BM, Jayaraman V, Laurent G (2006) Encoding and decoding of overlapping odor sequences. Neuron 51:467-482

119. Brown SL, Joseph J, Stopfer M (2005) Encoding a temporally structured stimulus with a temporally structured neural representation. Nat Neurosci 8:1568-1576
120. Backer A (2002) Pattern recognition in locust early olfactory circuits: priming, gain control and coding issues. http://resolver. caltech.edu/CaltechETD,etd-11212002-185244. Accessed 3 July 2008

121. Ditzen M, Evers JF, Galizia CG (2003) Odor similarity does not influence the time needed for odor processing. Chem Senses 28:781-789

122. Abraham NM, Spors H, Carleton A, Margrie TW, Kuner T, Schaefer AT (2004) Maintaining accuracy at the expense of speed: stimulus similarity defines odor discrimination time in mice. Neuron 44:865-876

123. Uchida N, Mainen ZF (2003) Speed and accuracy of olfactory discrimination in the rat. Nat Neurosci 6:1224-1229

124. Rinberg D, Koulakov A, Gelperin A (2006) Speed-accuracy tradeoff in olfaction. Neuron 51:351-358

125. Wesson DW, Carey RM, Verhagen JV, Wachowiak M (2008) Rapid encoding and perception of novel odors in the rat. PLoS Biol 6:e82

126. Guo J, Guo A (2005) Crossmodal interactions between olfactory and visual learning in Drosophila. Science 309:307-310

127. Duistermars BJ, Frye MA (2008) Crossmodal visual input for odor tracking during fly flight. Curr Biol 18:270-275

128. Szyszka P, Ditzen M, Galkin A, Galizia CG, Menzel R (2005) Sparsening and temporal sharpening of olfactory representations in the honeybee mushroom bodies. J Neurophysiol 94:3303-3313

129. Monod B, Mouly AM, Vigouroux M, Holley A (1981) La stimulation électrique multipolaire du bulbe olfactif, modèle d'étude du codage spatial dans le système olfactif. C R Seances Acad Sci III 293:731-734

130. Mouly AM, Holley A (1986) Perceptive properties of the multisite electrical microstimulation of the olfactory bulb in the rat. Behav Brain Res 21:1-12

131. Mouly AM, Vigouroux M, Holley A (1985) On the ability of rats to discriminate between microstimulations of the olfactory bulb in different locations. Behav Brain Res 17:45-58

132. Monod B, Mouly AM, Vigouroux M, Holley A (1989) An investigation of some temporal aspects of olfactory coding with the model of multi-site electrical stimulation of the olfactory bulb in the rat. Behav Brain Res 33:51-63

133. Cleland TA, Johnson BA, Leon M, Linster C (2007) Relational representation in the olfactory system. Proc Nat Acad Sci USA 104:1953-1958

134. Fleischmann A, Shykind BM, Sosulski DL, Franks KM, Glinka ME, Mei DF, Sun Y, Kirkland J, Mendelsohn M, Albers MW, Axel R (2008) Mice with a "monoclonal nose": perturbations in an olfactory map impair odor discrimination. Neuron 60:1068-1081

135. Yaksi E, Judkewitz B, Friedrich RW (2007) Topological reorganization of odor representations in the olfactory bulb. PLoS Biol 5:e178

136. Sato T, Hirono J, Tonoike M, Takebayashi M (1994) Tuning specificities to aliphatic odorants in mouse olfactory receptor neurons and their local distribution. J Neurophysiol 72:29802989

137. Malnic B, Hirono J, Sato T, Buck LB (1999) Combinatorial receptor codes for odors. Cell 96:713-723

138. Johnson BA, Leon M (2000) Modular glomerular representations of odorants in the rat olfactory bulb and the effects of stimulus concentration. J Comp Neurol 422:496-509

139. Johnson BA, Woo CC, Leon M (1998) Spatial coding of odorant features in the glomerular layer of the rat olfactory bulb. J Comp Neurol 393:457-471

140. Johnson BA, Ho SL, Xu Z, Yihan JS, Yip S, Hingco EE, Leon M (2002) Functional mapping of the rat olfactory bulb using diverse odorants reveals modular responses to functional groups and hydrocarbon structural features. J Comp Neurol 449:180-194 
141. MacLeod K, Bäcker A, Laurent G (1998) Who reads temporal information contained across synchronized and oscillatory spike trains? Nature 395:693-698

142. Turner GC, Bazhenov M, Laurent G (2008) Olfactory representations by Drosophila mushroom body neurons. J Neurophysiol 992:734-746

143. Hallem EA, Ho MG, Carlson JR (2004) The molecular basis of odor coding in the Drosophila antenna. Cell 117:965-979

144. Schlief ML, Wilson RI (2007) Olfactory processing and behavior downstream from highly selective receptor neurons. Nat Neurosci 10:623-630

145. Suh GS, Wong AM, Hergarden AC, Wang JW, Simon AF, Benzer S, Axel R, Anderson DJ (2004) A single population of olfactory sensory neurons mediates an innate avoidance behaviour in Drosophila. Nature 431:854-859

146. Sengupta P, Chou JH, Bargmann CI (1996) odr-10 encodes a seven transmembrane domain olfactory receptor required for responses to the odorant diacetyl. Cell 84:899-909

147. Wes PD, Bargmann CI (2001) C. elegans odour discrimination requires asymmetric diversity in olfactory neurons. Nature 410:698-701

148. Kobayakawa K, Kobayakawa R, Matsumoto H, Oka Y, Imai T, Ikawa M, Okabe M, Ikeda T, Itohara S, Kikusui T, Mori K, Sakano H (2007) Innate versus learned odour processing in the mouse olfactory bulb. Nature 450:503-508

149. Slotnick BM, Graham S, Laing DG, Bell GA (1987) Detection of propionic acid vapor by rats with lesions of olfactory bulb areas associated with high 2-DG uptake. Brain Res 417:343-346
150. Lu X-CM, Slotnick BM (1998) Olfaction in rats with extensive lesions of the olfactory bulbs, implications for odor coding. Neuroscience 84:849-866

151. Slotnick B, Cockerham R, Pickett EJ (2004) Olfaction in olfactory bulbectomized rats. Neurosci 24:9195-9200

152. DasGupta S, Waddell S (2008) Learned odor discrimination in Drosophila without combinatorial odor maps in the antennal lobe. Curr Biol 18:1668-1674

153. Benton R, Vannice KS, Gomez-Diaz C, Vosshall LB (2009) Variant ionotropic glutamate receptors as chemosensory receptors in Drosophila. Cell 136:149-162

154. Kashiwadani H, Sasaki YF, Uchida N, Mori K (1999) Synchronized oscillatory discharges of mitral/tufted cells with different molecular receptive ranges in the rabbit olfactory bulb. J Neurophysiol 82:1786-1792

155. Schoppa NE, Westbrook GL (2001) Glomerulus-specific synchronization of mitral cells in the olfactory bulb. Neuron 31:639-651

156. Schaefer AT, Margrie TW (2007) Spatiotemporal representations in the olfactory system. Trends Neurosci 30:10-92

157. Gollisch T, Meister M (2008) Rapid neural coding in the retina with relative spike latencies. Science 319:1108-1111

158. Rajan R, Clement JP, Bhalla US (2006) Rats smell in stereo. Science 311:666-670 